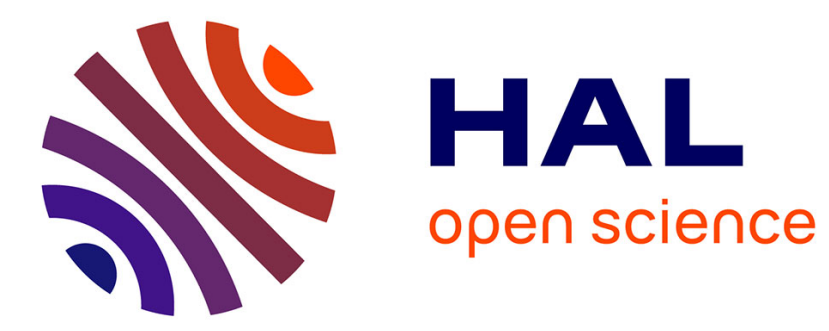

\title{
Tomographie ultrasonore par diffraction
}

B. Duchene, W. Tabbara

\section{- To cite this version:}

B. Duchene, W. Tabbara. Tomographie ultrasonore par diffraction. Revue de Physique Appliquée, 1985, 20 (6), pp.299-304. 10.1051/rphysap:01985002006029900 . jpa-00245336

\section{HAL Id: jpa-00245336 https://hal.science/jpa-00245336}

Submitted on 1 Jan 1985

HAL is a multi-disciplinary open access archive for the deposit and dissemination of scientific research documents, whether they are published or not. The documents may come from teaching and research institutions in France or abroad, or from public or private research centers.
L'archive ouverte pluridisciplinaire HAL, est destinée au dépôt et à la diffusion de documents scientifiques de niveau recherche, publiés ou non, émanant des établissements d'enseignement et de recherche français ou étrangers, des laboratoires publics ou privés. 
Classification

Physics Abstracts

$43.35-87.60 \mathrm{~B}$

\title{
Tomographie ultrasonore par diffraction
}

\author{
B. Duchene et W. Tabbara \\ Groupe d'Electromagnétisme, Laboratoire des Signaux et Systèmes, C.N.R.S.-E.S.E., Plateau du Moulon, \\ 91190 Gif sur Yvette, France
}

(Reçu le 4 octobre 1984, révisé le 21 décembre 1984, accepté le 4 février 1985)

\begin{abstract}
Résumé. - Nous décrivons ici un dispositif d'imagerie ultrasonore utilisant la méthode dite de "tomographie par diffraction ". Celle-ci permet de dresser la carte des sources secondaires induites dans l'objet par l'onde incidente, et ceci sans hypothèse a priori sur le mode de propagation de l'onde. Les caractéristiques du dispositif, ses performances et des exemples d'images de fantômes simples seront donnés. Parmi les utilisations possibles, nous présenterons les images, simulées, montrant la possibilité de détection des variations de température dans un processus d'hyperthermie.
\end{abstract}

\begin{abstract}
We present here in an ultrasound imaging set up based on the " Diffraction Tomography » approach. This method provides a map of the secondary sources induced in the object by the incident wave, without any a priori hypotheses on the wave propagation. The main features of the set up and examples of images of simple phantoms targets will be presented. Among possible applications, we show simulated images pointing out the possibility of detecting temperature changes in hyperthermia.
\end{abstract}

\section{Introduction.}

L'approche tomographique dans les problèmes de formation d'images ou de diagnostic de milieux inhomogènes, a trouvé des applications dans de nombreux domaines. Dans la majeure partie des utilisations de cette technique, les champs électromagnétiques et acoustiques sont supposés se propager suivant des trajets rectilignes. Cette hypothèse se vérifie bien avec les "Scanner-X ». Elle est à la base d'une nouvelle approche de l'échographie-B en ultrasons [1] où les champs sont mesurés en transmission et à fréquence fixe. L'hypothèse de la propagation rectiligne se vérifie bien dans le cas d'hétérogénéités du milieu petites devant la longueur d'onde ou peu diffringente. Ceci n'est pas souvent le cas dans les applications médicales des ultrasons où les phénomènes de diffraction sont importants et mettent en défaut l'hypothèse précédente [2]. Pour tenir compte des effets de diffraction l'approche tomographique a été modifiée par divers auteurs [3-5], mais ces formulations reposent sur une nouvelle simplification. Celle-ci consiste à considérer que l'objet est faiblement diffringent et en conséquence le champ ultrasonore en son intérieur égal au champ en l'absence de l'objet (approximations de Born et Rytov). Cette hypothèse est assez restrictive et il n'est pas aisé, pour des objets hétérogènes, de bien cerner son domaine de validité. Dans le cadre de ces approximations, de nombreuses études sont poursuivies pour mieux évaluer leurs performances et les conditions de leur utilisation [6-8]. Dans le cadre de ces approximations, l'image est la carte de $\left|k^{2}(\mathbf{x})-k_{0}^{2}\right|$ où $k(\mathbf{x})$ et $k_{0}$ représentent respectivement les nombres d'onde dans le milieu formant l'objet et celui du milieu extérieur. En acoustique on a $k(\mathbf{x})=\frac{\omega}{C(\mathbf{x})}+i \alpha(\mathbf{x}), \omega$ étant la pulsation de l'onde, $C(\mathbf{x})$ la vitesse du son dans le milieu considéré et $\alpha(\mathbf{x})$ l'atténuation dans ce milieu. Nous voyons immédiatement que la connaissance de $k^{2}(\mathbf{x})-k_{0}^{2}$ permet d'accéder à $C(\mathbf{x})$ et $\alpha(\mathbf{x})$ paramètres caractéristiques du milieu. Il faut aussi noter que dans la méthode décrite ci-dessus, on a négligé les variations de la densité $\rho(\mathbf{x})$ dans le milieu considéré, hypothèse raisonnable dans les applications médicales des ultrasons.

Afin de s'affranchir de la contrainte due à ces approximations et d'élargir le champ d'application de la méthode de tomographie par ultrasons, l'algorithme décrit dans [3] peut être repris intégralement sans y introduire d'approximations de type Born ou Rytov. Dans ce cas l'image obtenue est la carte du module de la fonction (en négligeant toujours les variations de densité) :

$$
J(\mathbf{x})=\left(k^{2}(\mathbf{x})-k_{0}^{2}\right) p(\mathbf{x})
$$

où $p(\mathbf{x})$ est le champ de pression dans le milieu formant l'objet. $J(\mathbf{x})$ représente alors la densité des sources 
induites dans l'objet par l'onde incidente. C'est cette adaptation de l'algorithme défini dans [3] qui a été utilisée dans le domaine microonde pour la réalisation d'un dispositif d'imagerie rapide $[9,10]$. Dans ce cas l'analyse a été développée pour un obstacle cylindrique, l'onde incidente ayant un champ électrique parallèle à l'axe oz du cylindre (polarisation $E$ ). L'équation (1) où $p(\mathbf{x})$ est remplacé par $E_{z}(\mathbf{x})$ est alors exacte.

Après cette modification il n'est plus possible d'accéder directement aux paramètres $C(\mathbf{x})$ et $\alpha(\mathbf{x})$, et il devient nécessaire de mettre au point une méthode permettant dans (1) d'extraire $\left(k^{2}(\mathbf{x})-k_{0}^{2}\right)$ de $J(\mathbf{x})$. Cette partie conduisant à une " imagerie quantitative " ne sera pas traitée ici.

Dans ce qui suit, nous décrivons la méthode permettant d'obtenir $J(\mathbf{x})$ à partir de la mesure du champ diffracté par l'objet. Nous présenterons les performances de cette méthode et nous examinerons la possibilité de son utilisation dans le contrôle de la température du milieu formant l'image.

\section{Dispositif expérimental.}

La figure 1 représente le dispositif expérimental utilisé dans cette étude. Il se compose d'un émetteur (E), un transducteur de $5 \mathrm{~cm}$ de diamètre travaillant à $2 \mathrm{MHz}$; d'un récepteur $(\mathrm{R})$, un "capteur aiguille" d'un diamètre de $0,6 \mathrm{~mm}$. Un mini-ordinateur HP1000 commande le déplacement de $\mathrm{R}$ suivant un axe horizontal (dispositif micro-control au $1 / 100 \mathrm{~mm}$ ) et commande la mesure du champ en amplitude et phase. L'objet (O) est solidaire d'un dispositif qui assure sa rotation autour d'un axe vertical (micro-control $1 / 100$ degré) et commandé par le calculateur. Emetteur, récepteur et objet sont plongés dans une cuve remplie d'eau.

L'acquisition des données se fait de la manière suivante. Pour une position donnée de l'objet, le capteur $\mathbf{R}$ relève les valeurs du champ en amplitude et phase en 64 points espacés de $\lambda / 2$ (le nombre de points est fonction de la capacité de l'ordinateur). Ces valeurs sont stockées et formeront les données pour la reconstruction d'une vue de l'objet. Ce dernier subit alors une rotation $\Delta \theta$ et le procédé précédent est répété pour l'acquisition des données de la deuxième vue. On peut ainsi mémoriser les données pour un nombre de vues fixé à l'avance. Le champ en l'absence de l'objet est aussi relevé aux mêmes points de mesure.

L'échantillonnage au pas de $\lambda / 2$ est nécessaire pour une bonne reconstruction de l'image. D'autre part, si $2 L$ est la longueur du segment de mesure de champ pour chaque vue et $D$ la distance de l'objet au centre de ce segment, ces deux quantités sont reliées par :

$$
\varphi=\operatorname{Arctan} \frac{L}{D} \gtrsim 45^{\circ}
$$

où $2 \varphi$ est l'angle sous lequel on observe le segment de mesure à partir du centre de l'objet.

Avec le déplacement mécanique du capteur $\mathrm{R}$, le temps d'acquisition de l'ensemble des données est lent

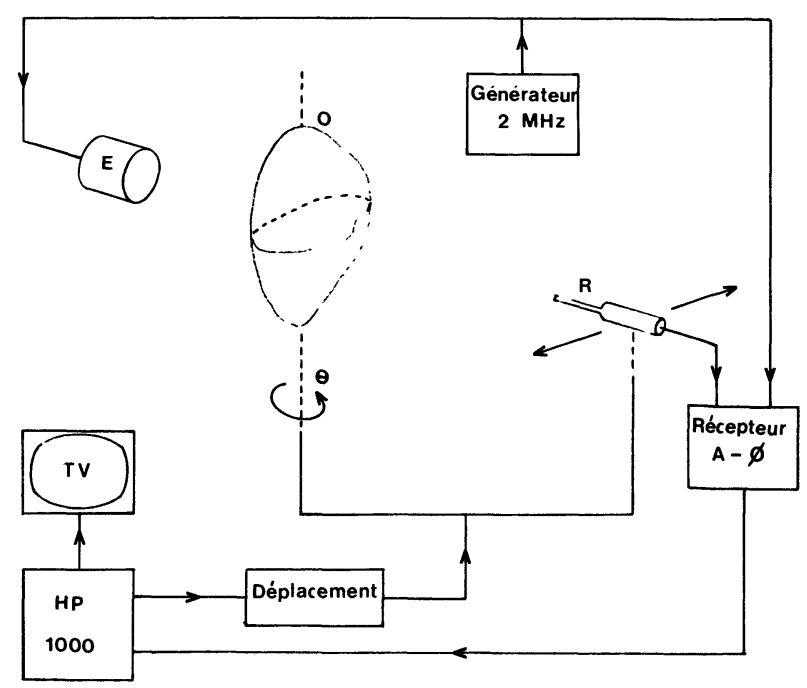

a)

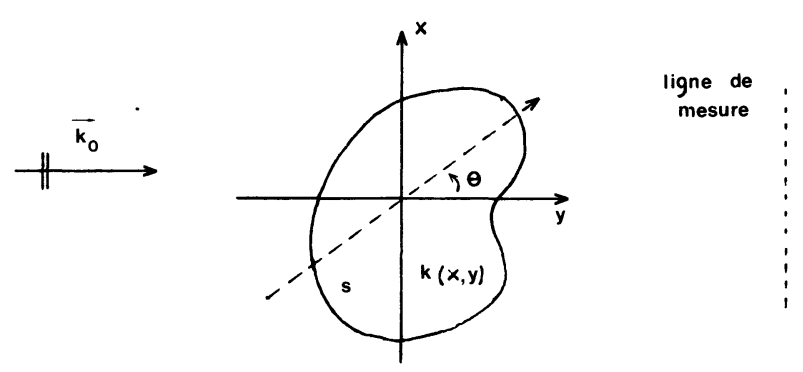

b)

Fig. 1. - (a) Schéma du montage; (b) Représentation dans le plan de la section reconstruite.

[(a) Experimental setup; (b) View in the plane of the reconstructed cross section.]

et des problèmes surgissent pour la mesure de la phase. Des procédures adéquates ont été mises en œuvre pour remédier à ces inconvénients et donner une bonne cohérence à l'ensemble des mesures. Le remplacement de $\mathrm{R}$ par une barrette de capteurs à balayage électronique est actuellement à l'étude avec le soutien technique de la CGR-Ultrasonic.

\section{Formation de l'image.}

L'image est une carte de paramètres caractéristiques de l'objet soumis au champ de l'émetteur, ou indépendant de ce champ. Le choix n'est certes pas unique et dépend généralemenţ de la modélisation de l'interaction de l'onde incidente avec l'objet. Nous avons choisi ici un modèle basé sur une représentation intégrale des champs et qui permet d'écrire :

$$
\begin{aligned}
p(\mathbf{x}) & =p_{0}(\mathbf{x})+\int_{\mathrm{s}} J\left(\mathbf{x}^{\prime}\right) G\left(\mathbf{x}, \mathbf{x}^{\prime}\right) \mathrm{d} \mathbf{x}^{\prime} \\
G\left(\mathbf{x}, \mathbf{x}^{\prime}\right) & =-\frac{i}{4} H_{0}^{2}\left(k_{0}\left|\mathbf{x}-\mathbf{x}^{\prime}\right|\right) \\
\mathbf{x} & =(x, y)
\end{aligned}
$$


où $p(\mathbf{x})$ représente le champ de pression mesuré par $\mathbf{R}$ en présence de l'objet, $p_{0}(\mathbf{x})$ celui en son absence, $H_{0}^{(2)}(t)$ la fonction de Hankel de seconde espèce et d'ordre zéro [11] et $\mathrm{S}$ la section droite de l'obstacle par un plan perpendiculaire à l'axe de rotation de celui-ci et contenant le segment de mesure. $J\left(\mathbf{x}^{\prime}\right)$ est la densité des sources induites donnée par (1). Cette modélisation suppose que l'obstacle est assimilable à un cylindre infini de section droite identique à S. Cette hypothèse est raisonnable dans la mesure où dans le dispositif utilisé, le capteur de réception est situé suffisamment près de l'objet. Dans ce cas les parties de l'objet contribuant au champ mesuré se situent dans une tranche cylindrique de faible épaisseur et de section droite S. Localement l'objet peut être assimilé à un cylindre infini. Cette hypothèse implicite dans [10] est confirmée de façon satisfaisante par les résultats expérimentaux obtenus ici et dans [10]. Dans la méthode utilisée ici, l'image est une représentation de la carte de $|J(\mathbf{x})| \cdot J(\mathbf{x})$ est une quantité complexe dont nous représenterons le module sous forme de teintes de gris. On pourrait aussi dresser la carte de la phase de $J(\mathbf{x})$, de sa partie réelle ou de sa partie imaginaire; chacune de ces quantités apporte une information différente sur l'hétérogénéité du milieu. Une étude systématique approfondie est encore nécessaire pour déterminer laquelle de ces quantités est la plus représentative de l'objet pour une application donnée.

Nous ne détaillerons pas ici l'analyse du processus de reconstruction, le lecteur intéressé pourra se reporter à la référence [10]. En bref, le schéma pour la formation d'une vue est le suivant :

1) A partir des mesures de $p(\mathbf{x})$ pour une position de l'objet, on définit les fonctions :

$$
\begin{aligned}
& \psi(\mathbf{x})=\frac{p(\mathbf{x})-p_{0}(\mathbf{x})}{p_{0}(\mathbf{x})} \\
& \varphi(\mathbf{x})=\frac{J(\mathbf{x})}{p_{0}(\mathbf{x})} .
\end{aligned}
$$

2) Soit $\hat{\psi}(\alpha, y)$ la transformée de Fourier de $\psi(x, y)$ par rapport à $x$ :

$$
\psi(x, y)=\frac{1}{2 \pi} \int_{-\infty}^{+\infty} \hat{\psi}(\alpha, y) \mathrm{e}^{-i \alpha x} \mathrm{~d} \alpha
$$

$y$ : ordonnée de la ligne de mesure.

3) On montre alors que :

$$
\begin{gathered}
\hat{\psi}(\alpha, y)=C(\alpha) \mathrm{e}^{-i\left(-k_{0}+\sqrt{k_{0}^{2}-\alpha^{2}}\right) y} \\
C(\alpha)=\frac{-i}{4 \pi \sqrt{k_{0}^{2}-\alpha^{2}}} \int_{\mathrm{S}} \varphi\left(\mathbf{x}^{\prime}\right) \mathrm{e}^{i \alpha x^{\prime}} \mathrm{e}^{i\left(-k_{0}+\sqrt{k_{0}^{2}-\alpha^{2}}\right) y^{\prime}} \mathrm{d} \mathbf{x}^{\prime} .
\end{gathered}
$$

A partir donc de $p(\mathbf{x})$ et $p_{0}(\mathbf{x})$ on peut calculer $C(\alpha)$ quantité directement reliée à $J(\mathbf{x})$ par l'intermédiaire de $\varphi\left(\mathbf{x}^{\prime}\right)$. Posons maintenant $\beta=-k_{0}+\sqrt{k_{0}^{2}-\alpha^{2}}$, on peut écrire :

$$
i 4 \pi \sqrt{k_{0}^{2}-\alpha^{2}} C(\alpha)=\int_{\mathrm{s}} \varphi\left(\mathbf{x}^{\prime}\right) \mathrm{e}^{i \alpha x^{\prime}+i \beta y^{\prime}} \mathrm{d} \mathbf{x}^{\prime}
$$

et l'on montre que ceci est vrai pour $(\alpha, \beta)$ tels que $\alpha^{2}+\left(\beta+k_{0}\right)^{2}=k_{0}^{2}$. $C(\alpha)$ est donc la restriction de la transformée de Fourier bidimensionnelle de $\varphi(\mathbf{x}) \mathbb{1}_{\mathbf{s}}(\mathbf{x}):$

$$
\begin{array}{rlrl}
\hat{\varphi}(\alpha, \beta) & =\iint_{-\infty}^{+\infty} \varphi(\mathbf{x}) \mathfrak{1}_{\mathbf{S}}(\mathbf{x}) \mathrm{e}^{i(\alpha x+\beta y)} \mathrm{d} x \mathrm{~d} y \\
\mathbb{1}_{\mathbf{S}}(\mathbf{x}) & =1 & \mathbf{x} \in \mathrm{S} \\
& =0 \quad \mathrm{x} \notin \mathrm{S}
\end{array}
$$

au demi-cercle de rayon $k_{0}$ centré au point $\left(0,-k_{0}\right)$ du plan spectral $(\alpha, \beta)$, figure 2. Pour obtenir $J(\mathbf{x})$ il faudrait pouvoir calculer la transformée de Fourier inverse de $\hat{\varphi}(\alpha, \beta)$, mais cette fonction n'est connue que pour $(\alpha, \beta)$ sur le demi-cercle et non dans tout le plan spectral. Pour « remplir ce dernier » on applique les relations (4) et (7) successivement aux données obtenues après une rotation $\Delta \theta$ de l'objet. Nous obtenons ainsi les valeurs de $\hat{\varphi}(\alpha, \beta)$ sur un nombre de demi-cercles égai au nombre de vues, figure 3 . Sur chaque arc de cercle nous avons 64 points obtenus à partir de (6) et du calcul de $\hat{\psi}(\alpha, y)$ par un algorithme de F.F.T.

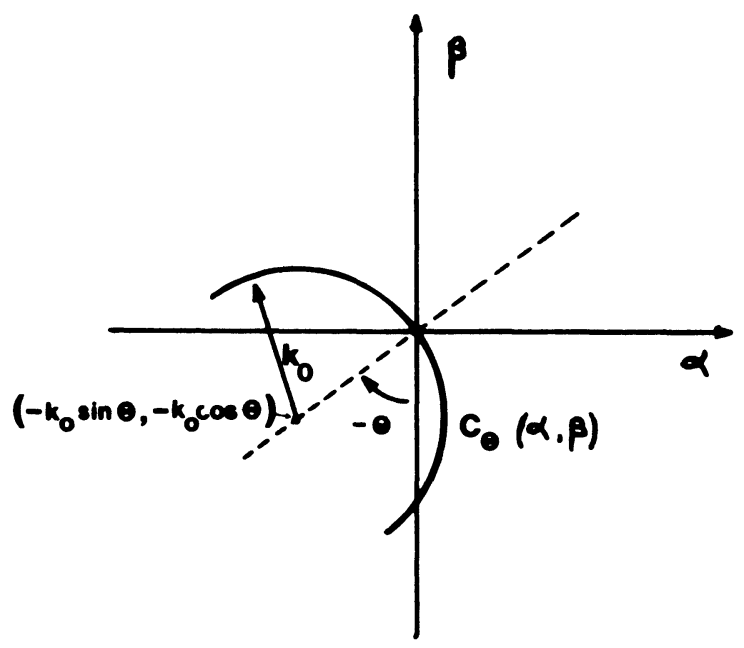

Fig. 2. - Lieu, dans le plan spectral, des points où les valeurs de $\varphi(\alpha, \beta)$ sont déterminées à partir des données d'une vue. Demi-cercle de centre $\left(-k_{0} \sin \theta,-k_{0} \cos \theta\right)$ et de rayon $k_{0}$.

[Locus, in the spectral plane, of the points where the values of $\varphi(\alpha, \beta)$ are determined from single view data. Half circle centred at $\left(-k_{0} \sin \theta,-k_{0} \cos \theta\right)$ and radius equal to $k_{0}$.]

4) Pour compléter le remplissage du plan spectral, nous calculons les valeurs de $\hat{\varphi}(\alpha, \beta)$ en des points situés en dehors des arcs de cercle par interpolation des valeurs obtenues sur ces arcs. Ceci nous permet 


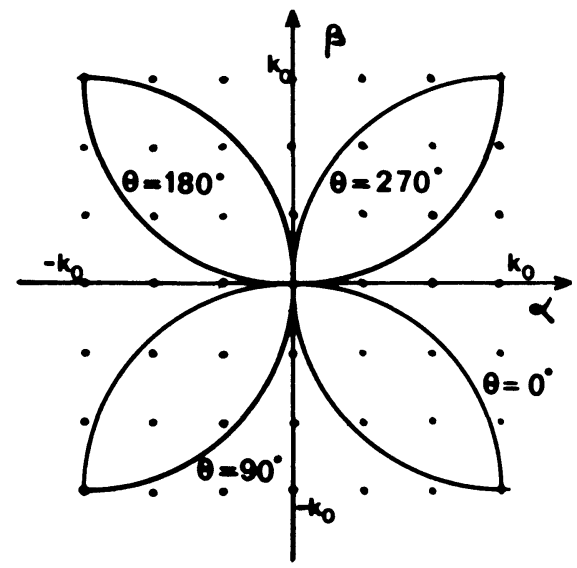

Fig. 3. - - Ensemble des points de $\varphi(\alpha, \beta)$ (4 vues) fournies par les données. • Points où $\varphi(\alpha, \beta)$ est obtenu par interpolation.

[ - Set of points where $\phi(\alpha, \beta)$ is computed from 4 views data. Points where $\varphi(\alpha, \beta)$ is obtained by interpolation.]

de former un maillage de points suffisant pour pouvoir calculer numériquement :

$$
\mathbb{1}_{\mathrm{S}}(\mathbf{x}) \varphi(\mathbf{x}) \approx \frac{1}{4 \pi^{2}} \iint_{-k_{0}}^{+k_{0}} \hat{\varphi}(\alpha, \beta) \mathrm{e}^{i(\alpha x+\beta y)} \mathrm{d} \alpha \mathrm{d} \beta
$$

et déduire $J(\mathbf{x})$ à partir de (4). Une interpolation précise (donc un nombre suffisant de points sur chaque arc) est nécessaire pour obtenir un résultat acceptable. L'augmentation du nombre de vues est évidemment un facteur important dans l'amélioration de la qualité de l'image.

Il faut noter que cette procédure ne remplit pas entièrement le plan spectral, mais seulement le carré centré à l'origine et de côtés de longueur $2 k_{0}$, l'extérieur du carré représentant la partie "évanescente» du spectre non accessible ici à la mesure. Théoriquement, des procédures de prolongement analytique permettraient d'accéder à l'information manquante, mais leur mise en ouvre est délicate et coûteuse en temps calcul. L'équation (9) représente en fait la convolution de $\vartheta_{\mathbf{s}}(\mathbf{x}) \varphi(\mathbf{x})$ par la transformée de Fourier de la fonction nulle en dehors du domaine carré défini ci-dessus et égale à 1 sur celui-ci.

\section{Résultats.}

La figure 4 représente l'image d'un fil de nylon de $0,6 \mathrm{~mm}$ de diamètre et constitue la réponse impulsionnelle du système d'imagerie. La figure $4 \mathrm{a}$ est l'image obtenue à partir d'une vue, c'est-à-dire en calculant $\varphi(\mathbf{x})$ à partir de $C(\alpha)$ au moyen de (6). Nous obtenons une bonne restitution de la dimension latérale du fil et un mauvais résultat dans le sens longitudinal. L'utilisation de 4 vues permet de corriger ce défaut et de fournir une image (4b) acceptable du fil. L'échelle

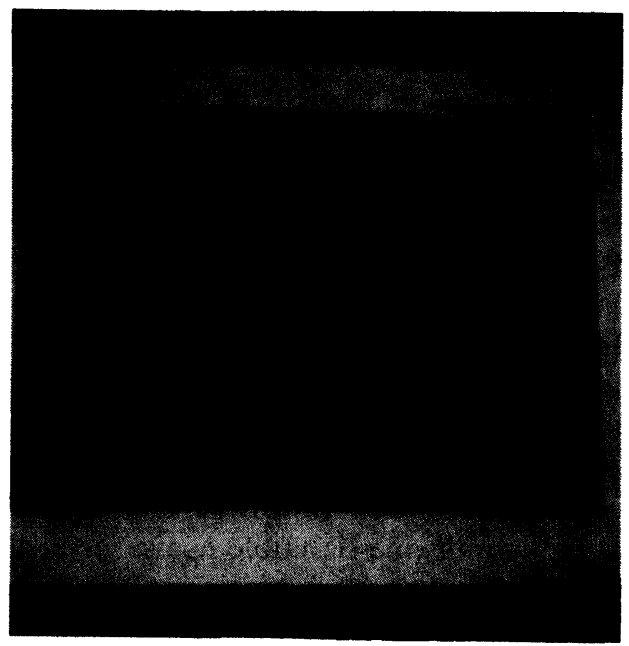

a)

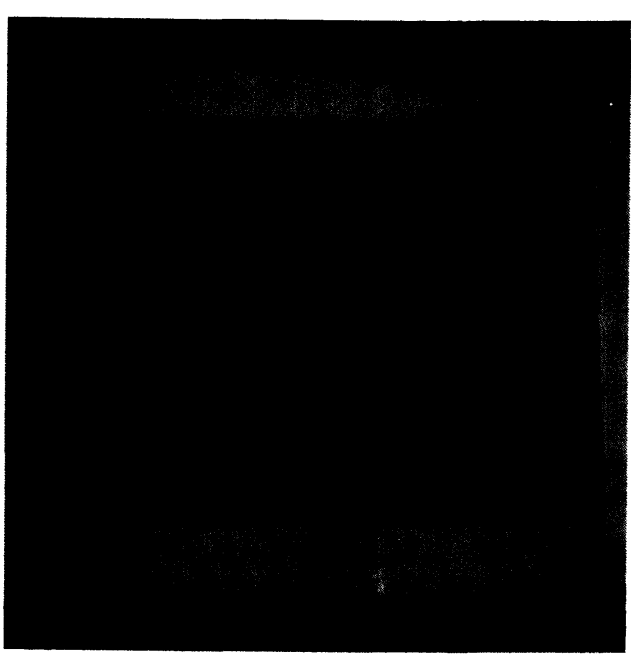

b)

Fig. 4. - Réponse impulsionnelle du système. Image d'un fil de nylon. $\varnothing 0,6 \mathrm{~mm}, f=2 \mathrm{MHz}$. (a) 1 vue; (b) 4 vues.

[Impulse response of the system. Image of a nylon string. $\varnothing 0.6 \mathrm{~mm}, f=2 \mathrm{MHz}$. (a) 1 view; (b) 4 views.]

sur le bord de l'écran ( $1 \mathrm{~mm}$ par division) permet d'apprécier la précision de la reconstruction.

La figure 5 représente l'image d'un tube de caoutchouc de diamètre intérieur égal à $3 \mathrm{~mm}$ et de diamètre extérieur égal à $5 \mathrm{~mm}$. En surimpression, les cercles noirs représentent les dimensions réelles du tube (le cercle extérieur n'apparaît pas, noyé dans le fond noir). Les dimensions du tube sont respectées. La figure $5 \mathrm{a}$ est obtenue à partir d'un seul relevé de champ utilisé pour les 4 vues, on a tenu compte de la symétrie du tube. Sur $5 b, 4$ vues mais avec 4 relevés différents; nous avons une bonne concordance avec 5a. Finalement $5 \mathrm{c}$ donne une image acceptable avec 8 vues, ce qui est peu; mais ceci tient aussi à la géométrie simple de l'objet.

La figure 6 représente une application possible de cette approche. Les images sont obtenues en simulant 


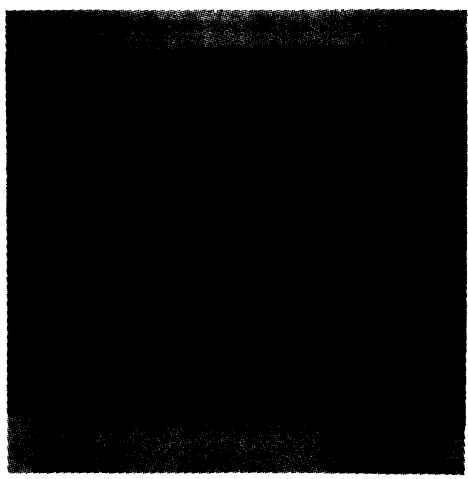

a)

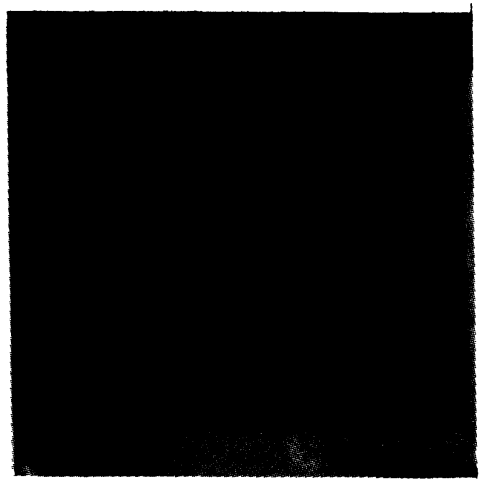

b)

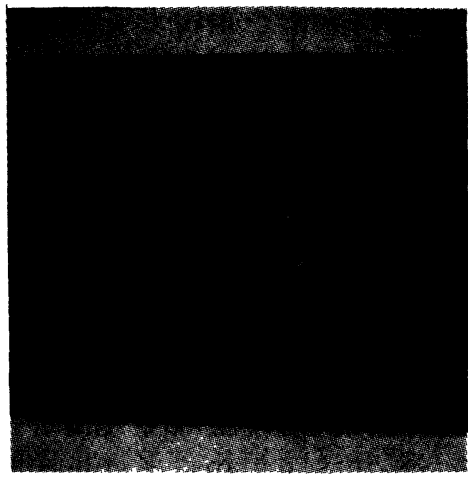

c)
Fig. 5. - Image d'un tube en caoutchouc naturel. $\varnothing_{\text {int }}=$ $3 \mathrm{~mm}, \varnothing_{\text {ext }}=5 \mathrm{~mm}, f=2 \mathrm{MHz}$. Contour réel du tube (contour extérieur noyé dans le fond noir). Echelle sur l'écran : $1 \mathrm{~mm} /$ division. (a) 4 vues et 1 relevé de champ. (b) 4 vues et 4 relevés de champ. (c) 8 vues et 1 relevé de champ.
[Image of a natural rubber pipe. $\varnothing_{\text {int }}=3 \mathrm{~mm}, \varnothing_{\text {ext }}=5 \mathrm{~mm}$, $f=2 \mathrm{MHz}$. - Exact geometry of the pipe (external contour undistinguishable from background). Scale on the screen : $1 \mathrm{~mm}$ /division. (a) 4 views from 1 set of field values. (b) 4 views from 4 sets of field values. (c) 8 views from 1 set of field values.]

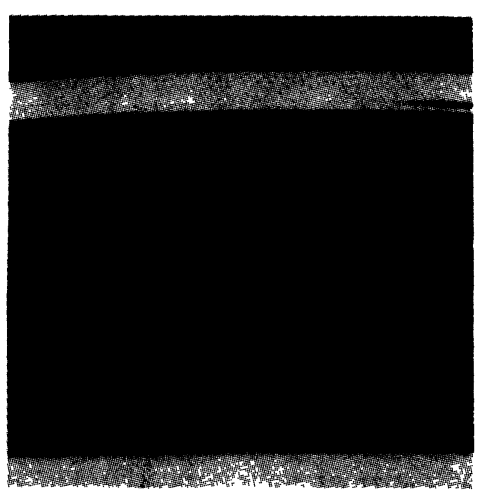

a)

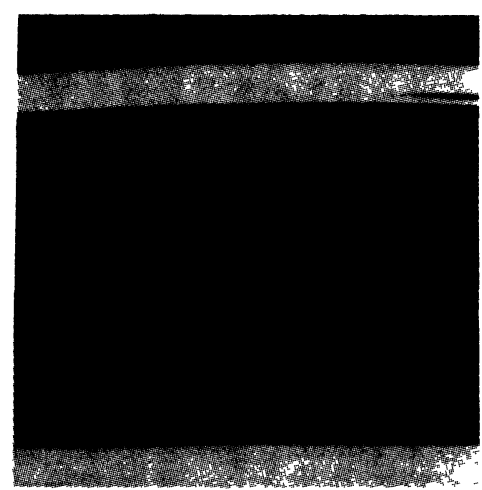

b)

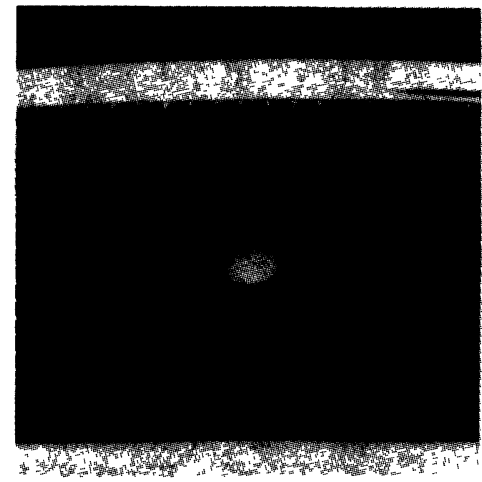

c)
Fig. 6. - Thermographie par imagerie différentielle. Tube en caoutchouc naturel, $\varnothing_{\text {int }}=3 \mathrm{~mm}, \varnothing_{\text {ext }}=5 \mathrm{~mm}$. Image de référence obtenue à $35^{\circ} \mathrm{C}$. (a) $37,5^{\circ}$; (b) $40^{\circ}$; (c) $42,5^{\circ}$.
[Thermography by differential imaging. Natural rubber pipe, $\varnothing_{\text {int }}=3 \mathrm{~mm}, \varnothing_{\text {ext }}=5 \mathrm{~mm}$. Reference image obtained at $35^{\circ}$ C. (a) $37.5^{\circ}$; (b) $40^{\circ}$; (c) $42.5^{\circ}$.] les mesures de champ diffracté. Celles-ci sont obtenues en résolvant de façon exacte le problème de l'interaction d'une onde plane avec le cylindre circulaire fluide [12]. Nous avons ici un tube de caoutchouc parcouru par de l'eau à des températures différentes. La vitesse du son dans l'eau est fonction de la température; comme cette vitesse apparaît dans $J(\mathbf{x})$ par l'intermédiaire du terme $k(\mathbf{x})$, toute variation de température entraîne un changement des valeurs de $J(\mathbf{x})$, donc une modification de l'image.

Nous avons réalisé une première image à la température de $35^{\circ} \mathrm{C}$, c'est l'image de référence. Celles représentées ici sont la différence entre l'image à la température $t$ et celle à la température de référence, d'où le nom d'imagerie différentielle. Nous remarquons une bonne mise en évidence des variations de température. Dans cet exemple, la variation de la célérité avec la température est donnée par la relation : $\Delta C / \Delta T=-2 \mathrm{~m} / \mathrm{s} /{ }^{\circ} \mathrm{C}$.

\section{Conclusion.}

Nous avons présenté ici une méthode simple et rigoureuse (aux variations de $\rho$ près qui ont été négligées) d'imagerie ultrasonore. Elle tient compte de tous les phénomènes d'interaction de l'onde incidente avec l'objet étudié. Elle permet pour des cibles simples une restitution précise de leur forme et de leurs dimensions.

Sont actuellement en cours, d'une part l'amélioration du dispositif de mesure par l'utilisation d'une barrette de capteurs et, d'autre part, l'étude de cibles complexes prélude à l'examen d'organes réels.

L'image obtenue ici est seulement qualitative. Il 
est intéressant à partir des valeurs de $J(\mathbf{x})$ de remonter aux paramètres physiques de la cible. Un moyen simple consiste à utiliser l'approximation du champ $p(\mathbf{x})$ dans l'obstacle par le champ incident $p_{0}(\mathbf{x})$, dans ce cas on a :

$$
\varphi(\mathbf{x})=\frac{J(\mathbf{x})}{p_{0}(\mathbf{x})}=\frac{\left(k^{2}(\mathbf{x})-k_{0}^{2}\right)}{p_{0}(\mathbf{x})} p_{0}(\mathbf{x})=k^{2}(\mathbf{x})-k_{0}^{2}
$$

d'où les valeurs de $k(\mathbf{x})$ donc de $C(\mathbf{x})$ et de $\alpha(\mathbf{x})$ la vitesse du son dans le milieu et l'atténuation de ce dernier. Cette approche a été utilisée dans [3] mais n'est malheureusement pas toujours valable et il est difficile de prévoir a priori quand elle l'est. Dans un prochain article nous présenterons une méthode rigoureuse permettant l'accès à $C(\mathbf{x})$ et $\alpha(\mathbf{x})$ à partir de la connaissance de $J(\mathbf{x})$ et nous préciserons les conditions de son utilisation.

La détermination de ces. paramètres physiques conduit à la mise en ouvre d'une « imagerie quantitative " permettant de caractériser le milieu étudié, et d'accéder à d'autres paramètres liés à $C(\mathbf{x})$ et $\alpha(\mathbf{x})$ comme la température.

\section{Bibliographie}

[1] Greenleaf, J. F., Gisvold, J. J., Bahn, R. C., A clinical prototype ultrasonic transmission tomographic scanner, in Acoustical Imaging 12, E. A. Ash and C. R. Hill Edit. (Plenum Press) 1982, p. 579-587.

[2] Devaney, A. J., A computer simulation study of diffraction tomography, IEEE Trans. Biomed. Engin. BME-30, no 7 (1983) p. 377-386.

[3] Mueller, R. K., KaVeh, M., Inverson, R. D., A new approach to acoustic tomography using diffraction techniques, in Acoustical Imaging 8, A. F. Metherell Edit. (Plenum Press) 1980, p. 615-628.

[4] DevaneY, A. J., A filtered backpropagation algorithm for diffraction tomography, Ultrasonic Imaging 4 (1982) 336-350.

[5] KaVeh, M., Soumekh, M., Mueller, R. K., A comparison of Born and Rytov approximations in acoustic tomography, in Acoustical Imaging $11 \mathrm{~J}$. P. Powers Edit. (Plenum Press) 1981, p. 325-335.

[6] Kaveh, M., SoumekH, M., Greenleaf, J. F., Signal processing for diffraction tomography, IEEE Trans. Sonics Ultrason., SU-31, no 4 (1984) 230-239.

[7] PAN, S. X., KAK, A. C., A computational study of reconstruction algorithms for diffraction tomography : interpolation versus filtered backpropagation, IEEE Trans. Acoust. Speech Sign. ASSP31, no 5 (1983) 1262-1275.
[8] Nahamoo, D., Pan, S. X., KaK, A. C., Synthetic aperture diffraction tomography and its interpolation-free computer implementation, IEEE Trans. Sonics Ultrason. SU-31, no 4 (1984) 218-229.

[9] Bolomey, J. Ch., IzAdNeGHadaR, A., JofFRe, L., Pichot, Ch., Peronnet, G., Solaimani, M., Microwave diffraction tomography for biomedical applications, IEEE Trans. Microw. Theory Techn. 30, no 11 (1982) 1998-2000.

[10] Pichot, Ch., Thèse Doctorat ès Sciences, Université Paris Sud, Orsay, avril 1982 (disponible auprès de M. Pichot, même adresse que les auteurs).

[11] Abramowithz, M., Stegun, A., Handbook of mathematical functions (Dover, New York) 1965.

[12] Duchene, B., TABbara, W., A geometrical optics method for assessing an inverse scattering problem for blood vessels-Part $1: A$ multistatic single frequency approach, IEEE Trans. Sonics Ultrason. SU-30 (1983) 13-19. 